\title{
Polymer Composites Filled with Multiwall Carbon Nanotubes
}

\author{
M. Kartel ${ }^{1,2}$, Yu. Sementsov ${ }^{1,2, *}$, S. Mahno ${ }^{2}$, V. Trachevskiy ${ }^{1,3}$, Wang Bo \\ ${ }^{1}$ Department of Architecture, Ningbo University of Technology, China \\ ${ }^{2}$ Chuiko Institute of Surface Chemistry, NAS of Ukraine, Ukraine \\ ${ }^{3}$ Institute of Transport Technologies, National Aviation University, Ukraine
}

Copyright $(2016$ by authors, all rights reserved. Authors agree that this article remains permanently open access under the terms of the Creative Commons Attribution License 4.0 International License

\begin{abstract}
The structural and physico-chemical characteristics of thermoplastic polymers filled with multiwall carbon nanotubes (CNTs) such as polyethylene (PE), polyamide 6 (PA 6) and layered fiberglass with PA 6 are investigated. The influence of their concentrations and homogeneity degree of nanotubes distribution is studied. The properties of new composites are compared with the well investigated polytetrafluoroethylene (PTFE)-CNTs and polypropylene (PP)-CNTs systems. It is shown that an addition of CNTs into thermoplastic polymeric materials leads to the significant changes in structural characteristics, growth of strength, electrical, thermal properties. It is coursed by the formation of CNTs continuous network in the original matrix, the crystallinity degree of the matrix depending on the concentration of CNTs. In turn, the crystallinity degree of the matrix is increased by homogeneity arising of the composite as a result of the strong interaction of the matrix with nanofiller. The changes of not only bulk but also the surface properties of the composites are observed, which explains the best biocompatibility of the nanocomposites observed in natural conditions experiments (in vivo).
\end{abstract}

Keywords Carbon Nanotubes, Polymers, Nanocomposites, Physical Properties

\section{Introduction}

New materials based on well-known matrixes filled with nanoparticles are under wide development in the field of materials science. During last decade, the new nanocomposites with multiwall carbon nanotubes (CNTs) as additives were extensively studied. CNTs are characterized by extremely high specific strength properties (tensile $\sim 1.8$ $\mathrm{TPa}$ ) [1], electrical and thermal conductivity. However, an unique property namely high aspect ratio $(\eta)$ (ratio of length to diameter), that is greater than $10^{3}$, is found for CNT distinguishing it from another nanoparticles. That is why, a percolation threshold $\left(\mathrm{F}_{\mathrm{c}}\right)\left(\mathrm{F}_{\mathrm{c}} \sim 1 / \eta\right)$, i.e. the concentration at which a continuous network of CNTs formed in the matrix of polymer at homogeneous distribution, can be equaled to $\sim 0.1 \%$ wt. $[2,3]$.

The goal of this study is to investigate the influence of the aspect number of nanoparticles and the homogeneity of nanoparticles distribution in the matrix on the properties of new composite polymer materials.

\section{Materials and Methods}

CNTs (fig. 1) was obtained by the CCVD (catalytic chemical vapor deposition method) by means of ethylene, propylene or propane - butane on mixed metal oxides catalysts with the ratio of metals $\mathrm{Al}_{2} \mathrm{Fe} / \mathrm{NiMo}_{0,21}$. The catalysts were obtained by coprecipitation or by evaporation of aerosol water-soluble salts of iron or nickel, aluminum and molybdenum [4-6].
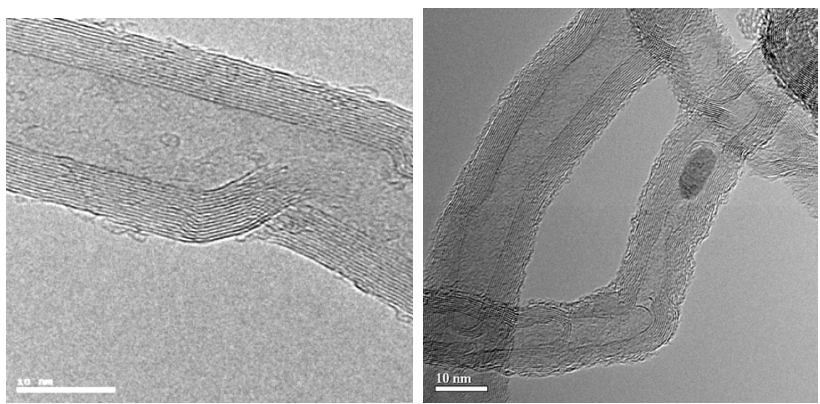

Figure 1. High resolutionTEM images of multiwall CNTs

CNTs chemical synthesis was carried out in the reactor with the volume $30 \mathrm{dm}^{3}$ where an yield of the product was about $1.5 \mathrm{~kg}$ per day. According to Ukrainian standard [7], the average diameter of CNTs was 10-20 nm and specific surface area determined by argon desorption was 200$400 \mathrm{~m}^{2} / \mathrm{g}$. Highly dispersed (pyrogenic) silica grade A-300 with a specific surface of $300 \mathrm{~m}^{2} / \mathrm{g}$ as a catalyst carrier was used to reduce agglomeration of CNTs leading to the 
loosening or dilution of the synthesized material. It allowed to obtain the product with the bulk density within 20-40 $\mathrm{g} / \mathrm{dm}^{3}$. For the purification of the synthesized materials from the mineral admixtures ( 8 to 18 wt. \%), a mixture of $\mathrm{NH}_{4} \mathrm{~F} \cdot \mathrm{HF}: \mathrm{H}_{2} \mathrm{O}: \mathrm{HCl}$ with the ratio of 150:1200:300 ml was used with the following rinsing with distilled water to neutral $\mathrm{pH}$. Pure CNTs were more agglomerated after drying and contained less than $1 \mathrm{wt}$. \% of mineral residual.

In particular, the agglomerates in the form of the entangled tubes with dimensions of 20-500 microns are obtained during an industrial CNT production applying CVD method [8, 9] (Fig. 2). While the composites filled with nanotubes possessing the extraordinary properties can be obtained due to their uniform distribution in the polymer matrix $[2,3]$. The design of the effective methods for the dispersion of CNTs agglomerates is necessary [10-12].

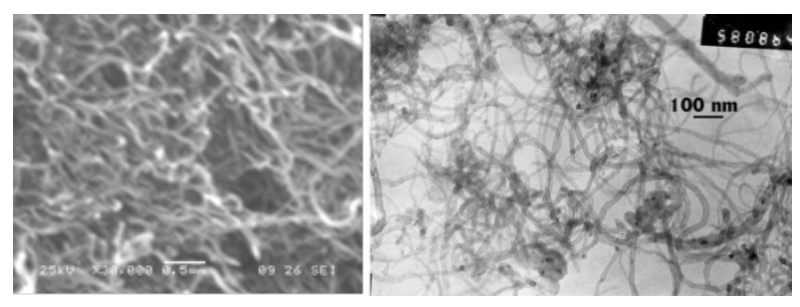

Figure 2. SEM and TEM images of multi-walled CNTs agglomerates

Deagglomeration (the decrease in the agglomerate size) of the synthesized product was carried out by ultrasound treatment (generator UZDN-2) or rotary homoginization that combines simultaneous effect of shear and cavitation mixing [13], in liquids (water, organic solvents, electrolytes, and others) in the presence of surfactants until the stable dispersion was formed. The next step was the "soft" mode mixing of a stable CNT dispersion with the polymer powder leading to more-or-less homogeneous distribution of the CNTS on the surface of the polymer particles. After drying, the samples were formatted by hot pressing.

According to the above mentioned procedure, the polyethylene - CNTs, polyamides 6 - CNTs, fiberglass - PA 6 - CNTs and PTFE with different content of CNTs systems were obtained. PTFE-CNTs system was also created after the coagulation of an aqueous dispersion PTFE 4D on the surface of CNTs. The dried powder mixtures with different contents of CNT $(0,05,0,1,1,0,2,0,5,0,10,0,15,0$ and 20.0 wt. \%) were shaped using hot pressing way. To determine the effect of the shape factor of nanofillers on the efficiency of polymer reinforcement, the systems consisting of PTFE filled with nanosized fumed silica (Aerosil) brand A300 (specific surface area $300 \mathrm{~m}^{2} / \mathrm{g}$ ) were also obtained $[14,15]$.

Nanocomposites based on PP brand 21060 with the content of CNTs in the range of 0.05 to 5.0 wt. \% were obtained by mixing $(50 \mathrm{rpm})$ the mixture of molten polypropylene and CNTs in the extruder. The initial samples were obtained in the form of granules that were further exposed to hot pressing [16-18].

Transmission electron microscopy (TEM) (JEM-100
CXII), scanning electron spectroscopy (SEM), JEOL SL6060LA, x-ray diffraction (XRD) (DRON-3M, $\lambda \mathrm{Co}=0,179 \mathrm{~nm}$ ), Raman scattering (CRS) (the spectrometer Brucker RFS 100/s, the radiation source is an argon laser, $\lambda=$ $514.5 \mathrm{~nm})$ were used to determine the structural characteristics of CNTs and polymer-CNTs composites. Structural and phase transformations as well as the processes connected with the destruction of polymer composites in air were investigated using differential thermal and gravimetric analyses (DTA, DTG) derivatograph Q 1500 D.

Dispersion of carbon nanotubes in different liquids was controlled by laser correlation spectroscopy (LCS) (spectrometer "ZetaSizer 3" from Malvern Instrument (UK) with correlation 7032 and a helium-neon laser LH-111 has a capacity of $25 \mathrm{~mW}$, wavelength $\lambda=633 \mathrm{~nm}$ ). Registration and statistical processing of laser radiation were performed for the 300-400 sec. Autocorrelation function was processed using the program PCS-Size mode v 1.61 [19].

Compression or tension tests of the polymeric materials and their composites was performed using tensile machine 2167-R50 with automatic recording of the deformation diagram.

Investigation of the surface of the initial PTFE and the nanocomposite PTFE-15\% CNTs was provided with an atomic force microscope NanoScope IIIa (Veeco Corp.) [17, 18]. The obtained data were processed using software GWIDDION. The surface of the samples were also examined with AFM with a probe modified by antibodies, immunoglobulin type $\mathrm{G}(\mathrm{IgG})$ obtained from animal serum.

Biocompatibility test of the samples was carried out by implantation of preformed materials in muscular pockets on the back of experimental animals.

\section{The Results and Discussion}

According to transmission electron microscopy (TEM) (Fig.1), X-ray diffraction, Raman spectroscopy data, different thermo gravimetric analysis (DTA, DTG), amorphous carbon was not detected.

Software [19] for LCS in the corresponding CONTIN mode ("modal field" approximation) allows to determine the distribution of particle volumes (in the case of constant density for the masses) and the number of particles of a certain size, in the approximation of spherical particles.

In Fig. 3-4 it is an example of a distribution of particle size for different systems which have been treated in a universal rotary homoginization.

Within this method, the system is considered to be "stable" or "almost stable" only when the distribution maximum is not shifted or shifted in different directions (left, right) after periodic shaking. If the shift occurs in one direction, the dispersions are considered as unstable. Thus, the water soluble surfactant - CNTs system (Fig. 3) is stable. Fig. 4: a) unstable, b)stable, g)"almost stable". 


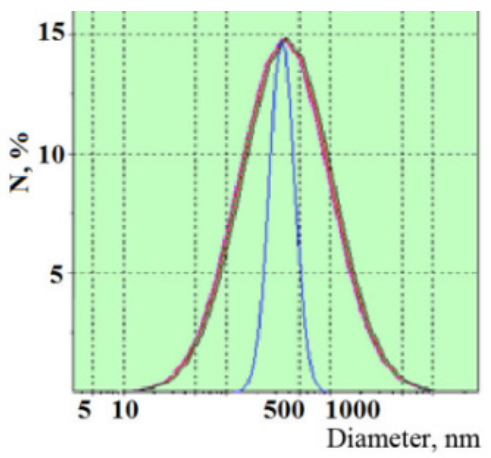

(a)

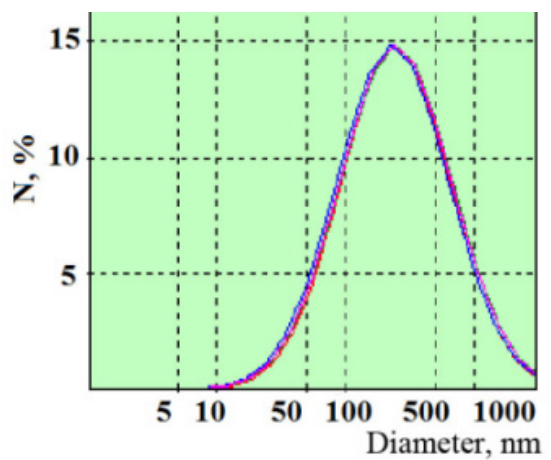

(b)

Figure 3. The particle size distribution of the "water-soluble polymer / surfactant / multiwall CNTs" system: as received (a) and one year later (b). Water soluble polymer - acrylic copolymer, surfactant - isodecyl alcohol ethoxylate.

It is concluded that the ultrasound treatment and rotary homoginization, which combines the action of shear deformation and cavitation mixing are effective method to obtain stable dispersions of CNTs; the stability of the dispersion and size of particles depends on the type of surfactant and concentration of the CNTs [20].

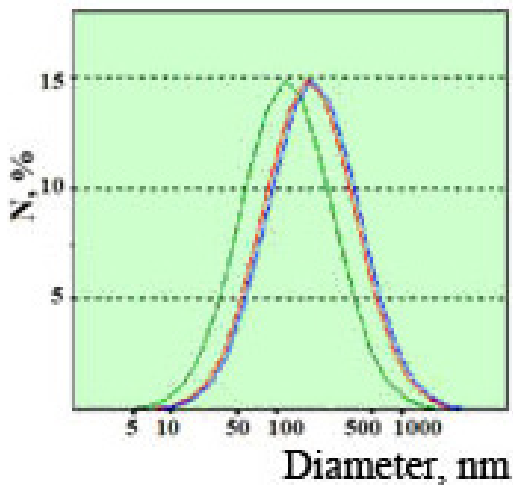

(a)

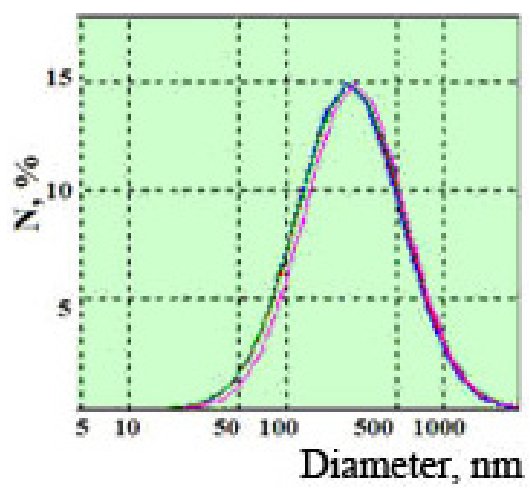

(c)

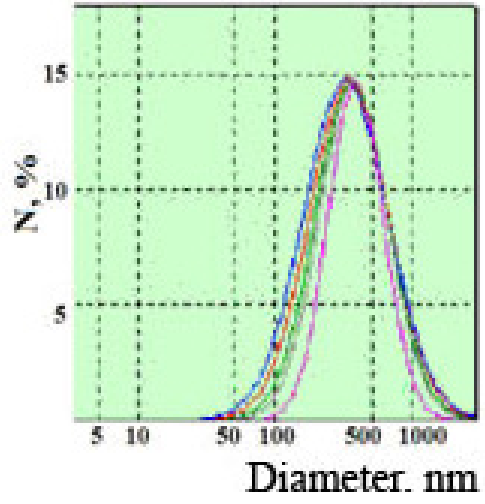

(b)

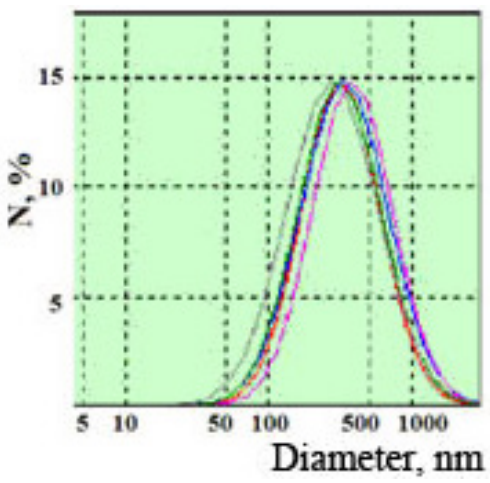

(d)

Figure 4. The particle size distribution in the "plasticizer-CNTs" system. An ionic surfactant - sulfonated melamin-formaldegide polycondensates was used as a plasticizer. The $4 \%$ aqueous solution of the plasticizer was dispersed in CNTs in the amount of $0 \%$ CNTs (a), $0.5 \%$ CNTs (b) $1 \%$ CNTs (c) and $1.5 \%$ CNTs (d). 


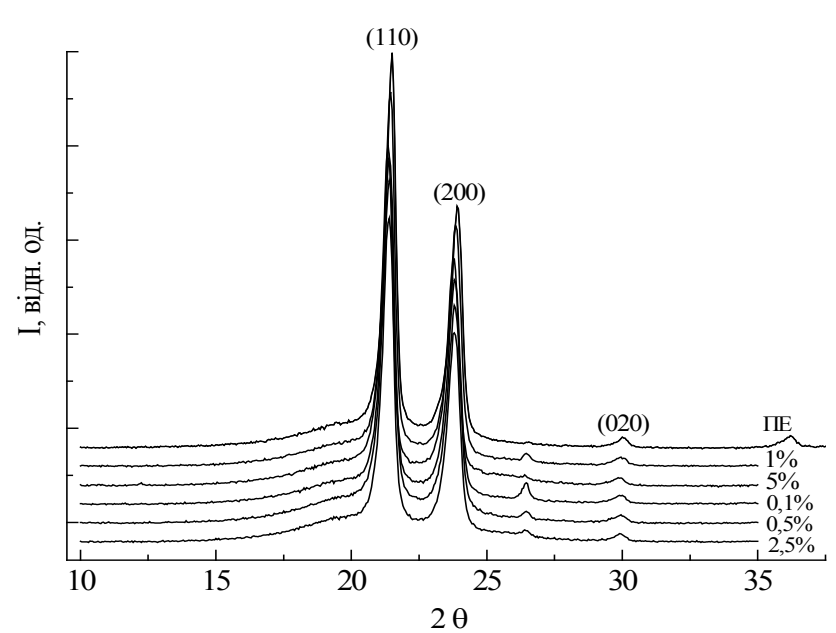

(a)

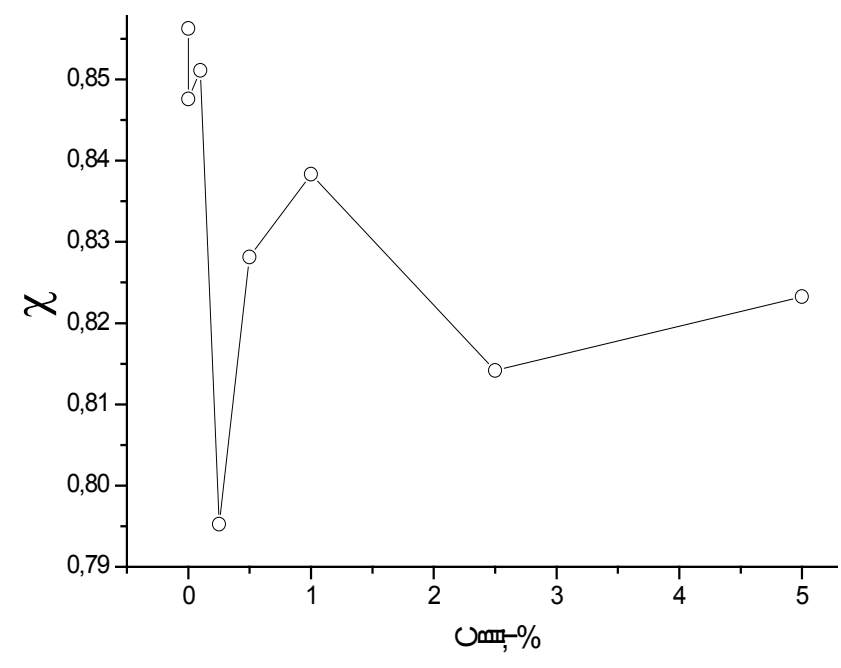

(b)

Figure 5. PE-CNTs system with the CNTs content from 0 to $2.5 \%$ wt.: a $\mathrm{x}$-ray diffraction; $\mathrm{b}$ - the dependence of the crystallinity degree $(\chi)$ on content of purified CNTs.

The structure parameters (crystallinity degree) of the obtained polymer - CNTs systems was determined from the profile analysis of $\mathrm{x}$-ray reflexes as shown in Fig. 5, showing the data for PE-CNTs system filled with the purified CNTS agglomerates.

The dependences of the crystallinity degree on the CNTs content for PE-CNTs systems with different pre-treatment of CNTs agglomerates are presented (Fig. 6). It follows that structural features begin at a lower concentration for a more dispersed CNTs. In the case of PE-CNTs composite from propane-butane (Fig. 6 a), the crystallinity degree for no purified CNTs is lowered when its the content is reached $0.05 \mathrm{wt} . \%$, while less change in the crystallinity degree is observed for purified one (the size of the agglomerates is greater) when its content becomes equal to $0.1 \mathrm{wt} \%$.

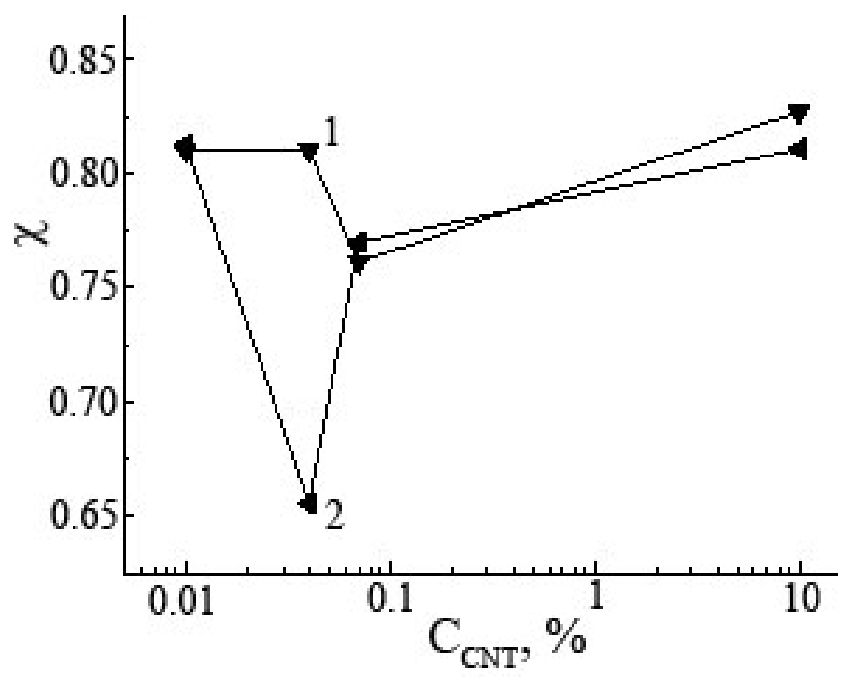

(a)

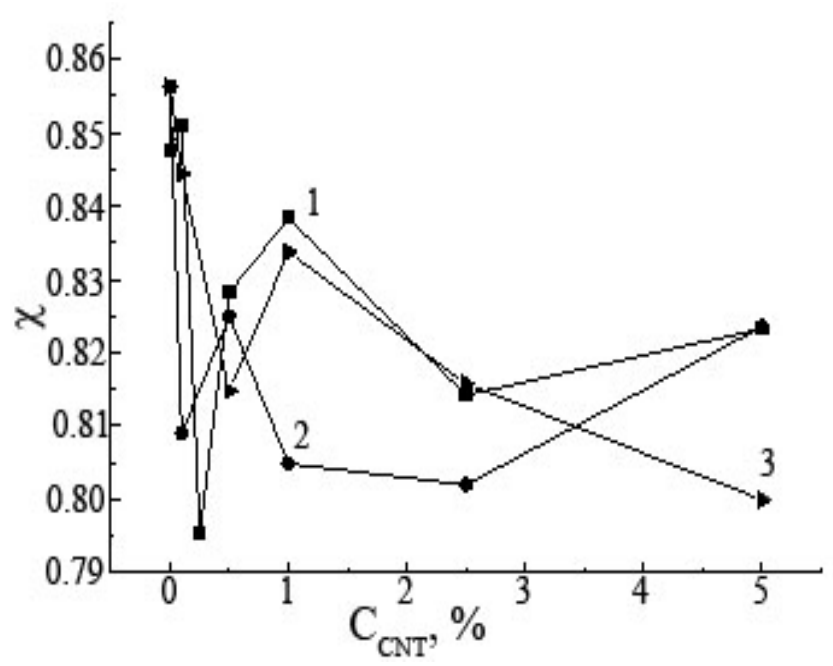

(b)

Figure 6. The crystallinity degree of PE-CNTs system vs CNTs content treated by various methods: a - CNTs of propane-butane 1 - purified, $2-$ no purified; $b-C N T s$ of propylene: 1 - purified, 2 - purified and disintegrated, $3-$ purified and treated with ultrasound.

For PE-CNTs system obtained from propylene (Fig. 6 b) at the minimum CNTs concentration, the greatest effect of the decrease in crystallinity degree is noted in the order: CNTs dispersed in the rotary homogenizer, CNTs dispersed by ultrasonic treatment and purified CNTs. The similar behavior is obtained for all the samples: the increase of the crystallinity degree at very low CNTs concentrations, then decrease, again increase, decrease and then gradual increase in degree of crystallinity. It is qualitatively correlated with results for systems PTFE-CNTs, PP-CNTs (Fig. 7-9) [14-18]. 

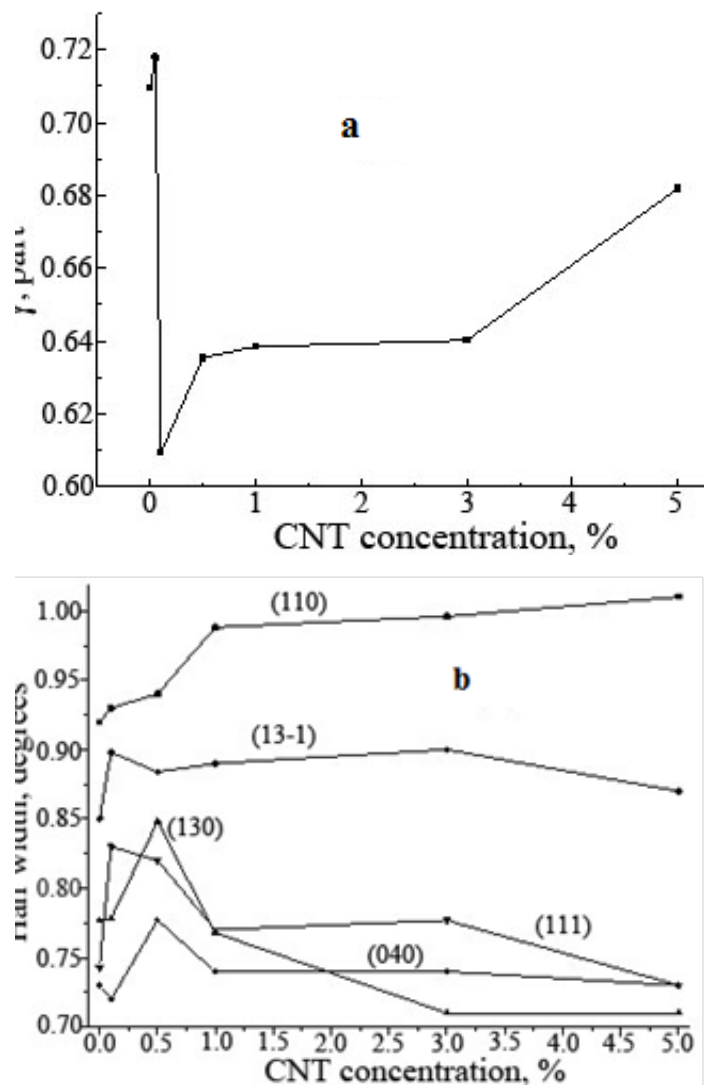

Figure 7. $\mathrm{a}-$ the dependence of the crystallinity degree determined from $\mathrm{X}$-ray on the concentration of carbon nanotubes in PP-CNTs system; b half-width dependence of X-ray reflexes on CNTs concentration in PPCNTs system

The result agrees well with the data from [21], where is the crystallization of polyethylene by shear strain in the presence of one-, five -, and multiwall carbon nanotubes leading to the changes in the structure of the PE matrix is shown. Distance between crystal planes (110) and (002) for the system of PECNTs is smaller than in the control sample of PE (without CNTs) and polymer chains are oriented along the axis of CNTs. The effect of CNTs on the formation of the structure of composites of $\beta$-propylene- ethylene copolymers is also noted herein [22].

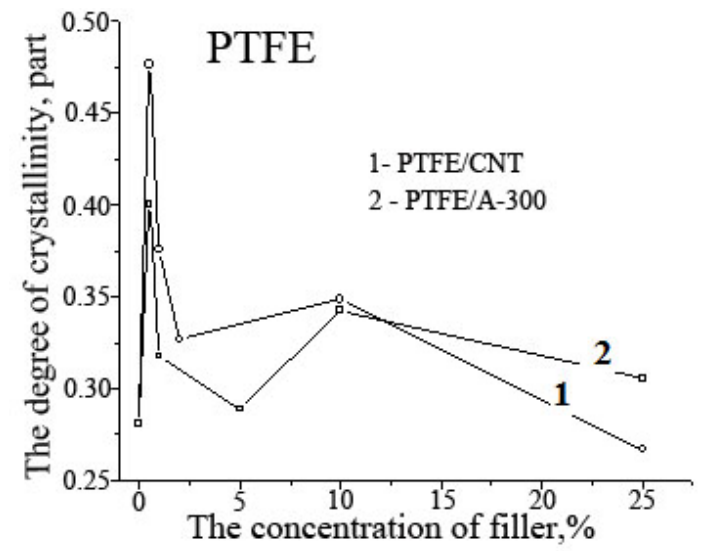

Figure 8. The dependence of the crystallinity degree of nanocomposites on filler concentration: 1 -PTFE/CNTs system; 2 -PTFE/ A-300 system.

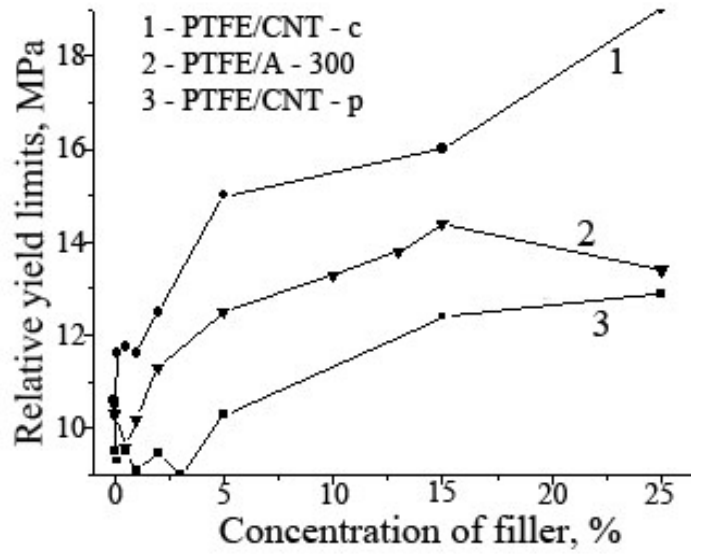

Figure 9. Dependence of relative yield limits on the concentration of filler for systems: 1 - PTFE/CNTs - cavitation; 2 - PTFE/A-300; 3 - PTFE/CNTs - powder.

The increase in the crystallinity degree at very low concentrations takes place due to the appearance of crystallization centers due to the presence of CNTs. When these centers begin to "interfere" each other, a number of "peripheral" molecules grow leading to the decrease of the size of the crystalline areas. This causes a growing tension at their boundary, as evidenced from the increase in the halfwidth of x-ray reflections (Fig. 7 for systems based on polypropylene). Further changes in crystallinity of the composite are associated with the formation of a continuous network of CNTs in the matrix polymer.

This is also confirmed by the results of DTA for system PE-CNTs (Fig. 10).

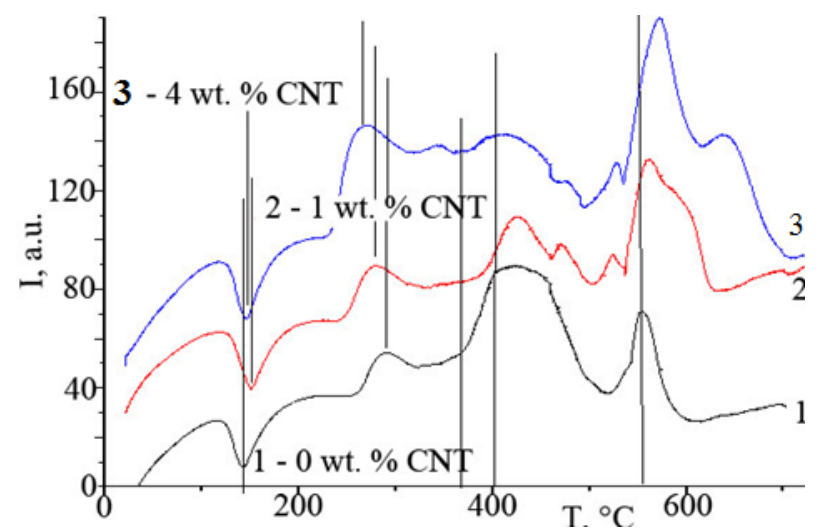

Figure 10. DTA results for PE-CNTs composites with different content of CNTs: $1-0 \%, 2-1 \%, 3-4 \%$

Fig. 10 shows that the temperature of endothermic peak melting point of the polymer is nonlinear depended on the content of CNTs and could indicate on a change in the crystallinity of PE and thermal conductivity of composites. The second exothermic peak that characterizes the removal of low molecular weight fractions from the polymer is shifted to the low temperature region by almost 20 degrees with increasing CNT content up to $4 \%$. So, the introduction of significant amounts of CNTs in the polymer can be a reason of the increase of low molecular fragments in the 
polymer. The temperature of the next peak of thermal oxidative degradation of the polymer increases with the increase of CNTs content to $2 \%$ almost on 60 degrees and decreases the total contribution to the energy of this process. Increasing the content of CNTs to $4 \%$, the temperature of transformation decreases and the energy contribution is growing up. With further temperature increase, the endothermic peaks are situated almost at the same temperature for all composites. The last DTA exothermic peak into a high-temperature region grows and becomes to be duplet at higher content of carbon nanotubes. It characterizes the temperature increase for degradation of polymer residuals and BHT. Thus, a relatively strong interaction between CNTs and polymer matrix resulting in the changes of the structure and properties of composite materials is confirmed.

A comparison of two nano-sized fillers of PTFE such as CNTs and Aerosil (Fig. 8,9) points on the great influence of the CNTs on the composite structure as well as its mechanical properties due to their larger aspect ratio. The highest homogenization of the CNTs using cavitation mixing of an aqueous emulsion PTFE with dispersion of CNTs (curve 1) is the most effective in compared with the mixing of powder PTFE and CNTs (curve 3) or filling with Aerosil (curve 2) as seen from the data of Fig. 8, 9. Similar behavior is appeared when the concentration dependency of elastic modulus and thermal conductivity for a system of PTFE and stress, strain and fracture conductivity of PP-CNTs system are considered $[14,15]$.

As can be seen, for all three systems, the degree of crystallinity varies considerably with the increasing content of CNTs. In addition, the more uniform distribution of CNTs in the matrix causes a sharp changes in structure and properties.

The mechanical characteristics of PA 6-CNTs system treated in the same manner are presented in table 1 . The loading in the coordinates "stress - strain" for PA 6 samples with different contents of CNTs are shown in Fig. 11.

Table 1. Tensile strength of PA6 - CNTS system vs. the CNTs concentrations.

\begin{tabular}{|c|c|c|c|c|}
\hline \multirow{2}{*}{$\begin{array}{c}\text { Properties for } \\
\text { tension }\end{array}$} & \multicolumn{4}{|c|}{ The concentration of CNT, wt.\%. } \\
\cline { 2 - 5 } & 0 & 0,125 & 0,25 & 0,50 \\
\hline $\begin{array}{c}\text { Tensile strength, } \\
\text { MPa }\end{array}$ & $25,0 \pm 1,2$ & $31,2 \pm 11,2$ & $41,2 \pm 1,0$ & $37,5 \pm 0,4$ \\
\hline $\begin{array}{c}\text { Deformation of } \\
\text { destruction, \% }\end{array}$ & 1,5 & 2,0 & 3,0 & 2,5 \\
\hline $\begin{array}{c}\text { Elasticity modulus, } \\
\text { MPa }\end{array}$ & 1700 & 2200 & 1900 & 1700 \\
\hline
\end{tabular}

As shown in table 1, the strength characteristics of the PA6-CNTS system exhibited non-linear dependence behavior, when CNTS is added into a polymer matrix. The maximum of the tensile strength and the maximum of destruction deformation are obtained for the composite containing $0.25 \mathrm{wt}$. $\%$ of CNTs.

The highest energy of deformation destruction (the area under the curve 3 in Fig. 11) is observed for the material of
PA6-0.125 wt $\%$ CNTS composition. In general, the increase of tensile strength leads to the decrease in fracture strain for elastic-plastic systems.

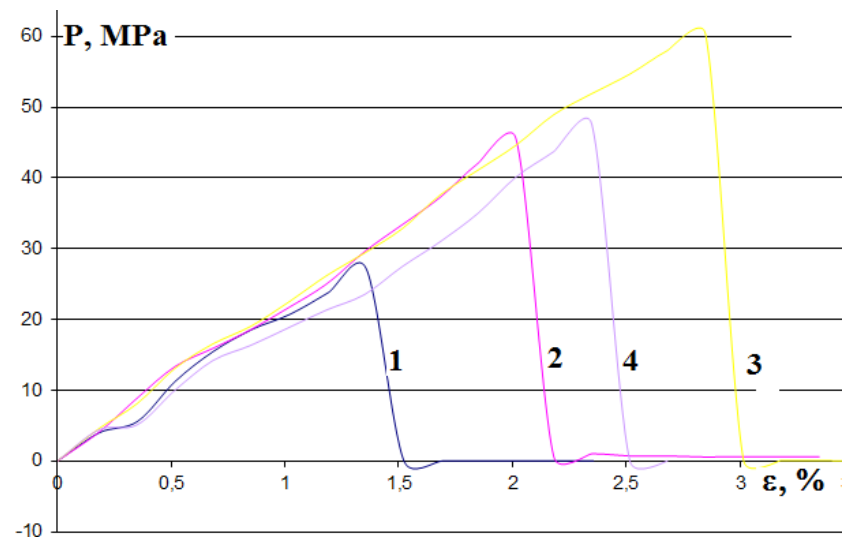

Figure 11. Dependence of tensile deformation on the tension system PA6-CNTS with the CNTS content, wt.\%: $1-0,2-0,125,3-0,25,4-$ 0,50 .

It follows that the destruction energy is slightly changed. We observe that the destruction energy of the PA 6-0.25 wt.\% CNTS composite is increased in $\sim 3$ times (!) in comparison with the initial polymer (PA 6-CNT) (Fig. 11). While, the highest value of elasticity module belongs to the PA 6-0.125 wt. \% CNTS system. The "stress - strain" curves for PA 6-CNT system have the typical for polymeric materials shape and are related to $\mathrm{V}$-type. A straight part of lines corresponds to the proportionality limit; the part of the line is corresponded to the deviation from Hooke's law connected with the segmental mobility of macromolecules, and the turnover limit after which the plastic deformation develops; the stages of hardening and fracture strain. However, the rebuilding of the structure (the "pulling balls") and the following strengthening are corresponded to at least two areas of plastic deformation of polymer (Fig. 11). Thus, the presence of CNTS in the amount up to $0.5 \mathrm{wt} . \%$ into the PA-6 matrix brings to the nonlinear increase of the strength characteristics of the composites comparing to the original one PA-6 (both of them were subjected to the same thermomechanical treatment). The highest tensile strength is noted for the PA 6-0.25 wt. \% CNTS composite.

Table 2. Characteristics tensile strength system consisting of three layers of fiberglass and two layers of PA 6: fiberglass - PA6 and the fiberglass PA6 -0.25 wt. $\%$ of CNTs.

\begin{tabular}{|c|c|c|c|}
\hline \multirow{2}{*}{ Sample } & \multicolumn{3}{|c|}{ Properties for tension } \\
\cline { 2 - 4 } & $\begin{array}{c}\text { Tensile strength, } \\
\text { MPa }\end{array}$ & $\begin{array}{c}\text { Deformation of } \\
\text { destruc- } \\
\text { tion, } \%\end{array}$ & $\begin{array}{c}\text { The modulus of } \\
\text { elasticity, MPa }\end{array}$ \\
\hline Fiberglass - PA6 & $209 \pm 6$ & $17 \pm 3$ & 1230 \\
\hline $\begin{array}{c}\text { Fiberglass - PA6-0.25 } \\
\text { wt. \% of CNTS. }\end{array}$ & $391 \pm 2$ & $12 \pm 1$ & 3255 \\
\hline
\end{tabular}

Thus, the introduction of 0.25 wt. \% of CNTS into PA6 that is used as a binder fiberglass leads to almost double increase in the tensile strength.

It is summarized that the changes in the structure and 
physical properties of the composites are caused by the strong interaction between CNTs and the polymer matrix.

The surface properties of the composites were studied using AFM [23]. The scheme of interaction AFM cantilever with the sample surface is shown in Fig. 12.

The force interaction between the probe and the surface (the magnitude of force "capture" and "separation", Fig. 12) was determined for PTFE and PTFE -15wt.\% CNTs nanocomposite in the gaseous media and in physiological solution $(0.9 \% \mathrm{NaCl})$ approximating to the system to natural conditions (in the body).

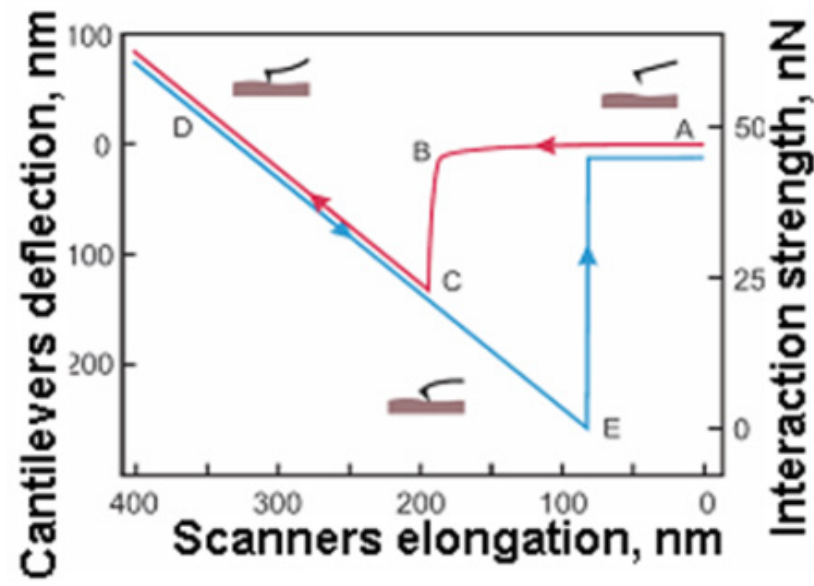

Figure 12. Diagram of electrostatic interaction cantilever with the sample surface. Curve A, B, C - "capture", curve D, E, - "pul-off".

An interaction "pure" probe and a probe modified with protein with surface nanocomposite much less in comparison with initial PTFE surface (Table 3).

Table 3. Force interaction AFM probe ("capture", "lead") with sample surface PTFE and PTFE-15\% CNTs

\begin{tabular}{|c|c|c|c|c|}
\hline \multirow{2}{*}{ Sample } & \multicolumn{2}{|c|}{ Air } & Water $(0.9 \% \mathrm{NaCl})$ \\
\cline { 2 - 5 } & Capture & Pul-off & Capture & Pul-off \\
\cline { 2 - 5 } & \multicolumn{4}{|c|}{ «clear» $\mathrm{Si}_{3} \mathrm{~N}_{4}$ probe / Force interaction, $\mathrm{nN}$} \\
\hline PTFE & $0.79 \pm 0.26$ & $10.64 \pm 0.33$ & $0.28 \pm 0.06$ & $3.72 \pm 0.22$ \\
\hline PTFE-CNT & $0.13 \pm 0.01$ & $8.32 \pm 0.38$ & $0.08 \pm 0.01$ & $0.92 \pm 0.06$ \\
\hline \multicolumn{5}{|c}{$\mathrm{Si}_{3} \mathrm{~N}_{4}+\mathrm{IgG}$ probe / Force interaction, $\mathrm{nN}$} \\
\hline PTFE & $2.37 \pm 0.22$ & $26.28 \pm 0.28$ & - & $1.82 \pm 0.06$ \\
\hline PTFE-CNT & $1.29 \pm 0.03$ & $9.63 \pm 0.21$ & - & $0.48 \pm 0.06$ \\
\hline
\end{tabular}

It was reported $[24,25]$ that the compatibility of artificial materials with living organism can be determined from the thickness of fibrous-connective capsule (pocket) formed around artificial material. The sample testing was carried out by means of an implantation of the preformed composite into the muscle "pouch" on the back of experimental animals In order to decrease the number of animals in the experiments and to receive the unbiased (more precise) results related to the organism reaction on the presence of the different artificial materials, the samples were implanted into the animal body in different parts of its back. Two types of samples as namely, PTFE (a) and PTFE filled with 15 wt. \% CNTs (b) were implanted in the same animal. In the case of PP-CNTs systems, the samples with different contents of CNTs $(0.05,1.0,3$, and 5 wt. \%) were implanted along both back's sides. Four weeks later, the samples with surrounding tissue were removed for the further histological analysis. The efficiency of body reaction was determined by measuring the thickness of fibrous-connective capsule formed around the sample. According to the histological data, the insertion of 15 wt.\% CNTs into PTFE matrix has an essential influence on the fibrous-connective formation around the sample. Morphometric of histological slice (Fig. 13) showed that the thickness of the formed capsule around PTFE was vissibly higher. For system PP-CNT, the lowest thickness of the capsule was fixed for $1.0 \mathrm{wt}$ \% CNTs in the matrix (Fig. 13). Hence, the presence of CNT in the matrix improves their biocompatibility.

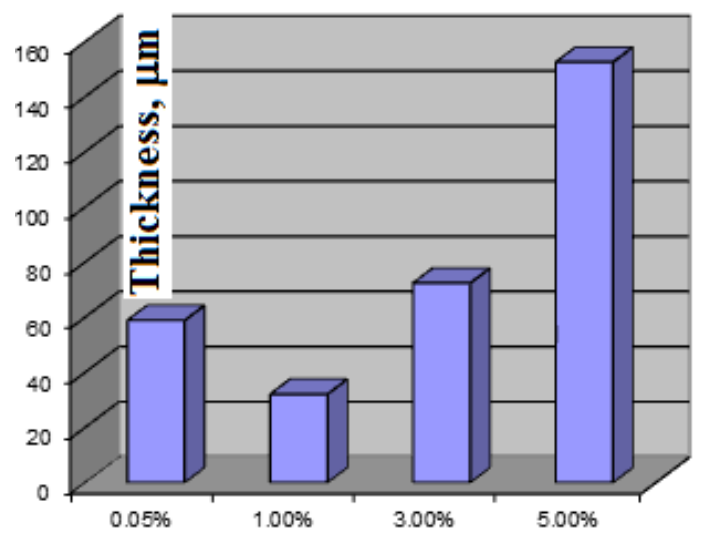

a)

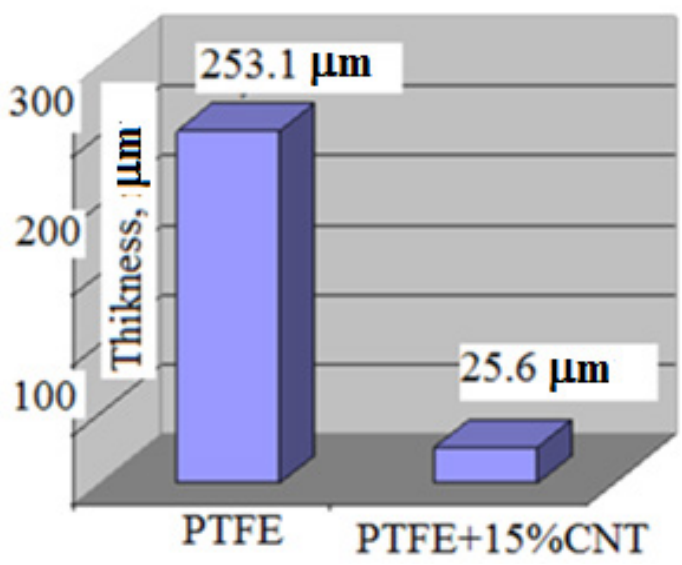

b)

Figure 13. Thickness of fibrous-connective formation around the sample: a) - system PP-CNTs; b) - PTFE-CNTs.

\section{Conclusions}

Carbon nanotubes are promising fillers for polymer materials due to their unique structure in conjunction with especial combination of strength, electrical and thermal properties. The reinforcing of polymer by CNTs even at low 
concentrations leads to the changes in both the bulk and surface properties of the material.

The improved biocompatibility of the filled polymers is observed for the modified surfaces as confirmed by experiments in vivo for PTFE-CNTs and PP-CNTs systems.

The change of the structural properties of the composite after CNTs introduction reflects the change of the melting temperature, the reduction of the degradation temperature of the low-ordered regions of the polymer and the increase of the degradation temperature of the crystalline regions of the composites indicating a relatively strong interaction of the CNTs with the polymer matrix.

The PA-CNTs system was advanced the recorded extremely high-performance stress and strain of the destruction increasing the destruction energy in 3-4 times in comparison with the original PA. It also shows that the strong interaction of CNTs with the matrix PA leads to the increase both the deformation and the tensile strain.

At the low CNTs concentration (below percolation threshold), the structural features were appeared as a result of the change in the ratio of crystalline and amorphous phases of the polymer. It is supposed that the amorphous phase appears around the nanopores,

The modified surface properties are responsible for the high biocompatibility of the filled polymers as demonstrated by in vivo experiments for PTFE-CNTs and PP-CNTs systems.

\section{REFERENCES}

[1] M.M.J. Treacy, T.W. Ebbesen, J.M. Gibson. Exceptionally high Young's modulus observed for individual carbon nanotubes, Nature, Vol. 381, 678-680, 1996.

[2] W. Bauhofer, J.Z. Kovacs. A review and analysis of electrical percolation in carbon nanotube polymer composites, Comp. Sci. Technol., Vol. 69, N10, 1486-1498, 2009.

[3] Bokobza L. Multiwall carbon nanotube elastomeric composites: A review, Polymer, Vol. 48, 4907- 4920, 2007.

[4] Sementsov Yu.I., Melezhek O.V., Prikhod'ko G.P. et al. Synthesis, structure, physico-chemical properties of nanocarbon materials. In: Shpak AP, Gorbyk PP (ed) Physical chemistry on nanomaterials and supramolecular structures, Kyiv: Naukova dumka, Vol.2, 116 - 158, 2007,

[5] Melezhyk A.V., Sementsov Yu.I., Yanchenko V.V. Synthesis of thin carbon nanotubes on co-precipitated metaloxide catalysts. Russian J. of Applied Chemistry, Vol. 78, N6, 938-946, 2005.

[6] Yanchenko V.V., Sementsov Yu.I., Melezhyk A.V. Method of obtaining of catalysts for CVD of carbon nanotubes. Ukrainian Pat. Application 20041008154, Int. Cl.7 C01B11/00, D01F9/12, 08.10.2004.

[7] CARBON NANOTUBES. Ukrainian Standard: TU U 24.1-03291669-009:2009 (ISC NAS of Ukraine).
[8] Hao Y., Qunfeng Z., Fei W. et al. Agglomerated CNTs synthesized in a fluidized bed reactor: Agglomerate structure and formation mechanism, Carbon, Vol. 41, 2855-2863, 2003.

[9] Wei F., Zhang Q., Qian W.-Z., et al. The mass production of carbon nanotubes using a nanoagglomerate fluidized bed reactor: A multiscale space-time analysis, Powder Technol., Vol.183, 10-20, 2008.

[10] Hessam Yazdania, Benjamin E. Smithb, Kianoosh Hatami Multi-walled carbon nanotube-filled polyvinyl chloride composites: Influence of processing method on dispersion quality, electrical conductivity and mechanical properties Composites Part A: Applied Science and Manufacturing Volume 82, March 2016, Pages 65-77

[11] Pat. USA, N7094467, DCA D 01 F 6/00, 2006. Antistatic polymer monofilament, method for making an antistatic polymer monofilament for production of spiral fabrics and spiral fabrics formed with such monofilaments / Zhang Heping, Harwood William, Ross Gilbert.

[12] Sokolov Y.A., Shubanov S.M., Kandyrin L.B., Kalugin E.V. Polymer nanocomposites. Structures. Properties, Plastics, N3, 18-23, 2009.

[13] Ovcharenko M. S. Influence of constructive and operating parameters on the energy characteristics of multi-function hydrodynamic unit - homogenizer: author. dis. for candidate of Sciences. the degree candidate. tech. Sciences: spec. 05.05.17 "Hydraulic machines and hydro-pneumo units" / M. S. Ovcharenko. - Amount 2013. - 23 p

[14] Sementsov Yu.I., Melezhyk A.V., Pyatkovsky M.L. et al. Properties of PTFE-MWNT Composite Materials / Hydrogen Materials Science and Chemistry of Carbon Na-nomaterials. NATO Security through Science. Series A: Chemistry and biology. Springer Science + Bussines Media, 757-763, 2007.

[15] Sementsov Yu., Prikhod'ko G., Kartel N. et al. Carbon nanotubes filled composite materials // In book :"Carbon Nanomaterials in Clean Energy Hydrogen Systems".P.183-195 NATO Science for Peace and Security Series C: Environmental Security. Zaginaichenko,S; Schur,D; Skorokhod, V, Veziroglu,A, Ibrahimoğlu,B (Eds.), Springer Science + Bussines Media B.V., 2011

[16] Resanova N.M., Kartel M.T., Sementsov Yu.I. et al. Rheological Properties of Molten Mixtures of Polypropylene Copolyamide/Carbon Nanotubes Mixture Melts // Chemistry, physics and technology of surface, Vol. 2, No4, 433-437, 2011.

[17] Sementsov Yu.I., Prikhod'ko G.P., Melezhyk A.V. et al. Physicochemical properties and biocompatibility of polymer/carbon nanotubes composites / In.book "Nanomaterials and Supramoltcular Structure". A.P.Shpak, P.P.Gorbyk (eds.). Springer Science + Bussines Media B.V., 347-368, 2009.

[18] Sementsov Yu., Gavriluk N., Prikhod'ko G. et al. Biocompatibility of Multiwall CNT and Nanocomposites on the Base of Polymers / In book:"Carbon Nanomaterials in Clean Energy Hydrogen Systems" NATO Science for Peace and Security Series C: Environmental Security. Bara-nowski,B; Zaginaichenko,S; Schur,D; Skorokhod, V, Veziroglu, A (Eds.), Springer Science + Bussines Media B.V., 327-334, 2008. 
[19] Scott E. McNeil. Characterization of Nanoparticles Intended for Drug Delivery, Humana Press, 2011, 283 p.

[20] E.O. Kovalska and Yu.I. Sementsov. Carbon nanotubes deagglomeration in aqueous solutions / In book «Nanomaterials Imaging Techniques, Surface Studies, and Applications»/ Springer Science + Bussines Media New York - 2013. - P. 61-72.

[21] Marilyn L. Minus, Han Gi Chae, Satish Kumar. Polyethylene Crystallization Nucleated by Carbon Nanotubes under Shear // ACS Appl. Mater. Interfaces.2012.- V. 4, iss.1.- P. 326-330.

[22] Dimitrios G. Papageorgioua, Lazaros Tzounisb, George Z. Papageorgiouc, Dimitrios N. Bikiarisc, Konstantinos Chrissafisa $\beta$-nucleated propylene-ethylene random copolymer filled with multi-walled carbon nanotubes: Mechanical, thermal and rheological properties Polymer Volume 55, Issue 16, 5 August 2014, Pages 3758-3769.

[23] Lazarenko O.M. and Lazarenko G.O. Method of individual testing of the implant compatibility with recipient's body, the Patent of Ukraine No. 87387 date of complaint date of publication of the Bulletin. No. 13, C2 A61B10/10 G01 N 33/53, 2009.

[24] Lacerda L., Bianco A., Prato M., Kostarelos K. Carbon nanotubes as nanomedicines: from toxicology to pharmacology. Adv Drug Del Rev, Vol. 58, 1460-1470, 2006

[25] Castner D.G., Ratner B.D. Biomedical surface science: foundations to frontiers. Surf Sci, Vol. 500, 28-60, 2002. 